\title{
"Dark Ages" in Comparative Studies and Ethnogenesis of Georgians in the Framework of the History of Comparative Studies
}

\author{
Tsira Baramidze \\ Institute of Caucasiology, Tbilisi State University, Georgia
}

Copyright $(2017$ by authors, all rights reserved. Authors agree that this article remains permanently open access under the terms of the Creative Commons Attribution License 4.0 International License

\begin{abstract}
The paper discusses the history of the issue of the ethnogenesis of Georgians. The comparative method of linguistics is universal and applicable to any language family. Like the methods of natural sciences the method is exact and verifiable. At some stages of contemporary linguistics, a crisis arose not because a method has some drawbacks, but because the method was considered to be useless to prove extralinguistic hypotheses by some linguists. As far as such extra-linguistic hypotheses cannot be proven by the comparative method, their authors tend to purposefully discredit comparative studies: I. They devalue the strictness of the method - language families are deliberately grouped into phyla; for instance, Noetic, Nostratic, Boreic, Dene-Caucasian... Hence, members of the Iberian-Caucasian family appeared in different phyla: Kartvelian languages - in Nostratic, Abkhaz-Adyghe and Daghestanian ones - in Dene-Caucasian. II. They ignore the method, linguistic items are qualified as languages and dialects by means of non-immanent marking. III. The comparative method is considered to be a non-universal one for the Iberian-Caucasian languages. IV. They consider the method to be unilateral, it was assumed that the method explained diverging process, however, it could not explain outcomes of convergence. V. The strength and credibility of sound correspondences was questioned; on the other hand, the establishment of secondary sound correspondences was considered possible. By way of a priori acknowledging the above-said, the allogenetic hypothesis was posited to be parallel with the theory of linguistic affinity. The paper analyzes the stages, having preceded the final proving of the Iberian-Caucasian affinity: it presents the research outcomes of the empirical data of the Iberian-Caucasian languages, the hologram principle of the reflection (resp. occurrence) of synchronic sound correspondences in diachrony, theoretical postulates determining the present-day level of the comparative study of the Iberian-Caucasian languages, research achievements and challenges. The detection of regular sound correspondences in the establishment of
\end{abstract}

language affinity is one and not the only objective of comparative studies. Another significant objective of comparative studies is the representation of the temporal-spatial model of the divergence of a parent-language of a family, the establishment of absolute chronology of the existence of a parent-language, the compilation of comparative phetics and grammars, etymological dictionaries, the localization of the homeland of the parent-language-speaking ethnic group, and the reconstruction of proto-culture. Attempts to establish "Dark Ages" in comparative studies periodically occur at various times with various data and make 'obstacles' to linguistics. The paper provides answers to the challenges in the framework of the comparative method. The ethnogenesis of Georgians is determined with respect to its belonging to the Iberian-Caucasian family.

Keywords Kartvelian Languages, Ethnogenesis, Comparative Linguistics, Sound Correspondences, Kinship, "Dark Ages"

\section{Introduction}

The issue of Kartvelian's ethnogenesis, or to which language family the Kartvelian languages belong has long and complicated history. The complex of this issue was caused by complicated character of the research object - the Caucasus - the mountain of languages. The Caucasian languages are grouped into three components: Iberian-Caucasian, Paleo-Caucasian and Areal Caucasian components. The Paleo-Caucasian component considers the relation with those ethnic groups, which created ancient civilization (Shumers, Chats, Hurrits, Pelazgs, Etruscs) and Basque language and culture. It is the truth that the areal of the Caucasian components (Turkish and Iranian) are millennial, but it is new in comparison with the rest of two 
(Iberian-Caucasian and Paleo-Caucasian) components. These components are migrated in the Caucasus in the various periods of the history. Those are Iranian and Turkish speaking ethnic groups. I don't discuss them at present, because the issue of ethnogenesis of Kartvelians - the problem of origin of the Georgian nation is intercrossed with the issue of the ethnoculture created by the Proto Caucasian or Iberian-Caucasian peoples in early millennia.

In linguistics the history of the ethnogenesis is connected with the name of Mari Brosset. Before him various researches were investigating the Caucasian languages (Stepano Paolini and Nikipore Irbachi, Fransisco Mario Majo, Sulkhan-Saba, Johan Giuldenschdedt, Julius Klaprot, Georg Rosen, Shora Nochma and others) (Chikobava 2008). But the putting the issue on the origin of the Caucasian languages belongs to Mari Brosset. The scholar represented the thesis according to which the Kartvelian languages belonged to the Indo-European ones. Frantz Bopp tried to confirm that Kartvelian languages were Indo-European ones.

An orientalist Anton Shiphner had never been at the Caucasus, but he created first scientific grammars of the mountain Iberian-Caucasian languages and he assisted Peter Uslar in his scientific work. During ten years (1861-1871) Uslar created 7 monographs. As the scholar considered "the greatest authority of the history of Georgia" Mari Brosset confirmed that the Iberian (Kartvelian) languages were Indo-European, but himself he doubted in his assertion. According to P. Uslar the issue of kinship of the Caucasian languages will be clear, when the relationship between the Caucasian and Iberian languages groups are researched (Chikobava 2008).

On the starting stage of the Caucasian languages studies $\mathrm{P}$. Uslar undoubtedly considered that this was one language family - Caucasian languages family. Researches of the Abkhazian, Chechen and four Daghestanian basic languages revealed the genetic unity of the North Caucasian languages indisputably. P. Uslar clearly saw the difference among the pre-scientific comparison and comparison of the historical linguistics.

Max Muller represented the thesis of the kinship of the Caucasian languages with Turanian ones. He could offer any scholarly argument. Friedrich Muller tried to confirm that the Caucasian languages were isolated ones and lately he himself doubted in his assertion.

The Caucasian languages were researched by other scholars (Richard Erkert, Adolf Dirr, Niko Marr and others) but the issue of genesis of Caucasian languages had a priori disposition for a long time because they tried to confirm the genesis without historical-comparative method.

Ivane Javakhishvili and Arnold Chikobava made a great contribution in researching the Kartvelian genesis. To settle this question they gave priority to morphological structure and noted that researching of the phonetics had an essential role to settle the question of genesis (Javakhisvili 1937, Chikobava 1979).

A comparative method of linguistics is as an exact and verified as the methods of natural sciences.

Any science is competent within the own limits, outside of which there is a sphere of non-verified hypotheses.

A comparative method of linguistics is universal and equally useful for any family of languages.

The crisis which was created in some parts of the modern comparative linguistics is caused not by the gaps of uselessness for confirming the hypotheses of one part of linguistics, simple of extra-linguists.

Impossibility of confirming some scholars' hypotheses through comparative method discredited it: they united language families into the phyla. The members of the family of the Noetic, Nostratic, Boreic, Dene-Caucasian and others, in particular, Iberian-Caucasian languages are united in two different phylas: the Kartvelian languages in the Nostratic (together with Indo-European, Semitic, Vralian, Altaian and Dravidian) and Abkhaz-Adyghe and Nakh-Dagestanian ones - in the Dene-Caucasian (together with Sino-Tibetian, Yeniseian and Na-dene languages of American Indians) phylas (Kurdiani 2007).

\section{1. "Science cannot endure the pointlessness"}

Why do I still speak about this fashion Nostratic theory? A reason is simple: In the soviet linguistic literature Georgian was included into Nostratic languages, the Abkhaz-Adyghe, Nakh, Daghestanian languages have not been attributed to any circle of the languages up-to-date.

Speaking about the kinship of the Kartvelian languages with Nostratic ones not any author said a word in favor of the contact of the North-Caucasian languages with the South-Caucasian ones. It comes out, that the Kartvelian languages can be linked to the Indo-European, Dravidian languages, but they cannot be linked to the North-Caucasian ones.

Science approaching here is poor. This is just that pointlessness which science cannot endure.

At present, through correct and adequate using of a historical-comparative method the kinship of the Kartvelian and mountain Caucasian languages is finally and clearly confirmed, also the kinship of Basque language with these languages is substantiated and a place of Basque in the family of the Iberian-Caucasian languages is defined. Two fundamental works of the latest period give me base to say this. These are: Merab Chukhua's monograph "Comparative grammar of the Iberian-Ichkerian languages (2008) and Mikheil Kurdiani's "Fundamentals of the Iberian-Caucasian linguistics" (2007).

In 1954-1956 in the journal "Linguistic issues" it was carried out discussion on the issues of internal and external kinship of the Iberian-Caucasian languages. Simon Janashia's hypothesis of external kinship meant the existence of the family of so-called Khetian-Iberian languages, in which along with the Iberian-Caucasian languages, non Indo-European and non-Semitic ones (Hatti, Urartu, Hurrit, Elam and others) of Minor and Front Asia 
were considered. The macro family's idea was created in the $19^{\text {th }}$ century (Nenorman, Hommel...). In that period they were called the "Alarodian languages". But the kinship of any of these dead languages with the Iberian-Caucasian family or with its any group has not been confirmed till present day. This is the issue of Paleo-Caucasian linguistics.

The principle outcome of the discussion, for which this discussion had been inspired, strengthened a nihilistic attitude towards the inner kinship of the Iberian-Caucasian languages among the scholars of other languages' family who could not obtain the Kartvelian and mountain Caucasian languages data. One group of scholars' states: it has not been verified up-to-date; it will not be ever verified. But at this time in the linguistic Caucasiology there were some sound-correspondences studied by Rene Lafon (Basque-Kartvelian, 1945), Togo Gudava (Daghestanian-Kartvelian, 1954) and Ketevan Lomtatidze (Abkhaz-Kartvelian, 1955). Though they cannot be sufficient for true, obvious and undoubtedly confirmation of the kinship of the Iberian-Caucasian languages, but the facts should be taken into account in confirming this kinship.

Unfortunately, the supporters of a strict scientific method, who had nihilistic attitude towards the fact that there were not many attempts of confirming the kinship of the Iberian-Caucasian languages, for some reason forgot the demand of obligation of defending a methodological strictness, when the Kartvelian languages, let's say, were considered to be undoubted members of the Nostratic languages' family together with the Semitic-Hamit, Indo-European, Uralian, Dravid and Altaian languages by Vladislav Ilich-Svitich (1934-1966). The Kartvelian languages' data was used for creating a Nostratic hypothesis incorrectly and incompetently.
The similar picture is obvious, e.g. in relation with the Semito-Hamit (Levin 1995) and Dravid (Andronov 1982) languages data (an outstanding Turkologist-comparativist, A.M. Shcherbak had a serious claim on this hypothesis).

A.R. Bomhard tried to specify so-called Nostratic "sound-correspondences" established by Ilich-Svitich, but actually he did it based on the same material and the result, naturally appeared to be analogous, though the formula of "sound-correspondences" were different (Bomhard 1990).

Holgar Pedersen attributed the Kartvelian languages to the "Nostratic languages" with six language families of the ancient world in one phylum: Semitic-Hamitic, Indo-European, Vralian, Dravidian, Altaian, Kartvelian.

At the boundary of the $20^{\text {th }}-21^{\text {st }}$ cc. a number of such fantastic theories just on kinship among the families of languages, is large (D. Worth, K. Ulenbeck, K. Bouda, E. Levy, S. Starostin, Comrie).

S. Starostin went on too long: he "confirmed" the kinship of the North-Caucasian and Yeniseian languages (Starostin 1982: 149-237) through "discovering the sound-correspondences" among them, then he united them to one phylum together with Sino-Tibetian languages, of course, through "establishing such sound-correspondences".

Discussing the phyla, it is essential to have trustworthy compared material and the etymological dictionary. But at present we have none of them.

A catastrophic picture of ignoring the strict scientific method in the modern linguistics, its general tendency, is clearly seen on the example of a scientific-popular journal "Atlas of the world languages" (an editor of which is an outstanding caucasiologist Bernar Comrie) in which such genealogic scheme is given:

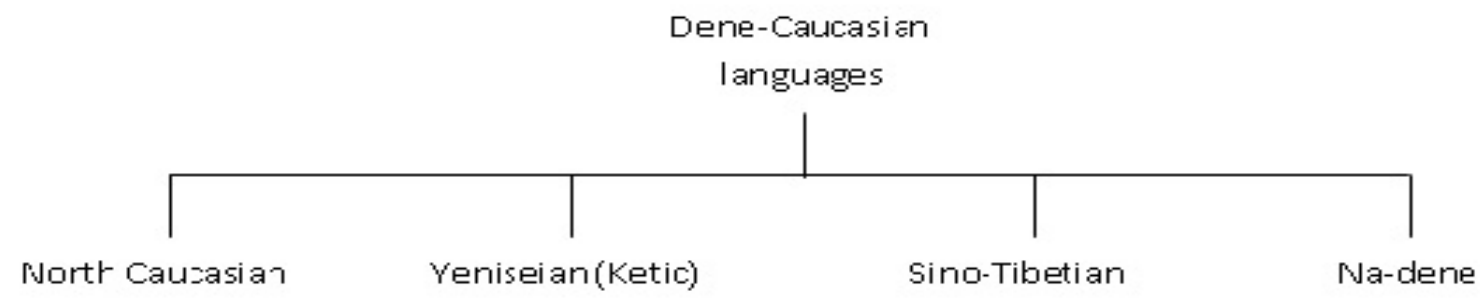


The idea is created and implanted, that the kinship of the North-Caucasian and South-Caucasian languages with any language family, but not with each other is confirmed or supposed. Their unity is supposed in any phylum, but not in same one (criticism on including the Iberian-Caucasian languages in different phylums see Khalidov 2006: 21-26).

Nikolay Trubetskoy expressed his skepticism in relation with the confirming the kinship of the supposed members of so-called "big language families" of phylums (1933), but later (1987) Viach. Ivanov considered, that "there is a large lexical material in Ilich-Svitich's dictionary of the Westratic languages, which gives right to be avoided Trubetskoy's this essential answer". (Trubetskoy 1987).

Maybe in future the regular sound-correspondences among them will be confirmed but today the existence of any language phylum is not scientifically confirmed.

In 1979, when this fateful danger for linguistics was not still clear, in the preface of the "Introduction to the Ibero-Caucasian linguistics" Arn. Chikobava wrote: "Great diversity is characteristic for the modern theoretic linguistics in understanding the essential issues".

Everybody "rings from his/her bell-tower", so that it is unclear what he/she "rings" and for what he/she "rings". Vagueness in these issues causes much harm in scientific studying of the Iberian-Caucasian languages in past (Chikobava 1979).

A scholar implied those which were created by N. Marr's Japhetic theory, for general linguistics. As it is well-known, the first period (1908-1916) includes the Japhetic languages, in particular, the Kartvelian languages or Iberian languages, some dead languages of the Front Asia belong to this circle, the second period (1916-1920) - the North Caucasian and Japhetic languages, i.e. this is a Caucasiologic period. In the third period (1920-1923) Japhetic circle passes the limits of the Caucasus and covers the Mediterranean space (Pelazgian and Etruskian). N. Marr put forward a new principle of static development, he opposed genealogic classification to stadia one.

Confirming the kinship of the North-Caucasian languages (in which almost nobody doubted) is connected with $\mathrm{N}$. Trubetskoy. G. Dumezil and A. Abdokov who researched the comparative grammar of the North-Caucasian languages, but not regular phonetic sound-correspondences.

Creating the historical-comparative morphology of the kindred languages is purposeful if there is a historical-comparative phonetics of these languages, i.e. their kinship is clearly confirmed via revealing a system of natural and regular sound-correspondences.

Some researches compare the North-Caucasian (Abkhaz-Adyghe and Nakh-Daghestanian) languages with the South-Caucasian (Kartvelian) languages, considering, as if their inter-kinship is more trustworthy or is confirmed, then their kinship with the South-Caucasian languages.

In Mikheil Kurdiani's monograph "Fundamentals of the Iberian-Caucasian linguistics" it is clearly confirmed that the North-Caucasian (Abkhaz-Adyghe and Nakh-Daghestanian) languages are not so genetically closer to each other, then each of them with the South-Caucasian (Basque-Kartvelian) languages.

In 1985 Viacheslav A. Chirikba published an article "The Basque and South-Caucasian languages" (Chirikba 1985: 95-105), which was harshly, though objectively criticized and was considered to be a fantastic sphere (Trask 1995: $81-86$.$) .$

The works by Bergston, Ruhlen, Starostin and Chirikba can be attributed to the fantastic sphere, as well.

The kinship of the Basque-Kartvelian and mountain Caucasian languages and the fact that they belong to one Iberian-Caucasian family has already been confirmed via those strict and verified methods, through which the kinship of the Indo-European languages is confirmed. The hypotheses of the Nostratic and Dene-Caucasian phyla according to which the South and North Caucasian languages belong to principally different genealogical trees has no rational ground (all the more a place of Basque just in a Basque-Kartvelian group of the Iberian-Caucasian language family was clearly established by Ian Brown and Mikheil Kurdiani).

In a historical-comparative method the verifying, testing of research outcomes which defines degree of its trustworthiness is essential.

Verification has three stages: a) material verification; b) system verification and c) typological verification.

Landmark work on Caucasian studies "Fundamentals of the Iberian-Caucasian languages" by Mikheil Kurdiani represents the criticism of the western scholars' attitude and method in confirming the kinship of the Iberian-Caucasian languages. Among the researchers of the Iberian-Caucasian languages - especially among the Georgian researchers a historical-comparative method was an object of discussion to specify these languages. Owing to this such examples were brought which were considered to be satisfied using the method correctly: phonetic processes - comparative, positive, superlative or acknowledging a parent-language as a reflex of different phonemes, or borrowings and others.

The monograph discusses the past stages till final confirming of kinship of the Iberian-Caucasian languages.

The monograph shows the correction of traditional researching priorities of Georgian linguistic school - from comparative morphology of the Iberian-Caucasian languages till phonetics, generalization, perfecting of traditional, canonical, theoretical basis of comparative method, rising the degree of verification of research outcomes relevantly with the strictness of method and defending profanation of a method from the following process. This process is operated just by genetic kinship of the North-Caucasian languages.

The monograph discusses many phonetic laws and rules established in the empiric researching process of the Iberian-Caucasian languages. The monograph explains certain phonetic phenomena, defines formula of sound-correspondences within the language groups, a root 
structure of the Iberian-Caucasian languages and stem-forming models, fully functional and material identity of these models in the South and North Caucasian languages, the principles of reflecting the synchronic sound-correspondences in diachrony.
The monograph represents a core paradigm of non-identical sound-correspondences on the basis of what it was possible to confirm the kinship of the Iberian-Caucasian languages:

\section{Sound Correspondences in the Kartvelian and Daghestanian Languages:}

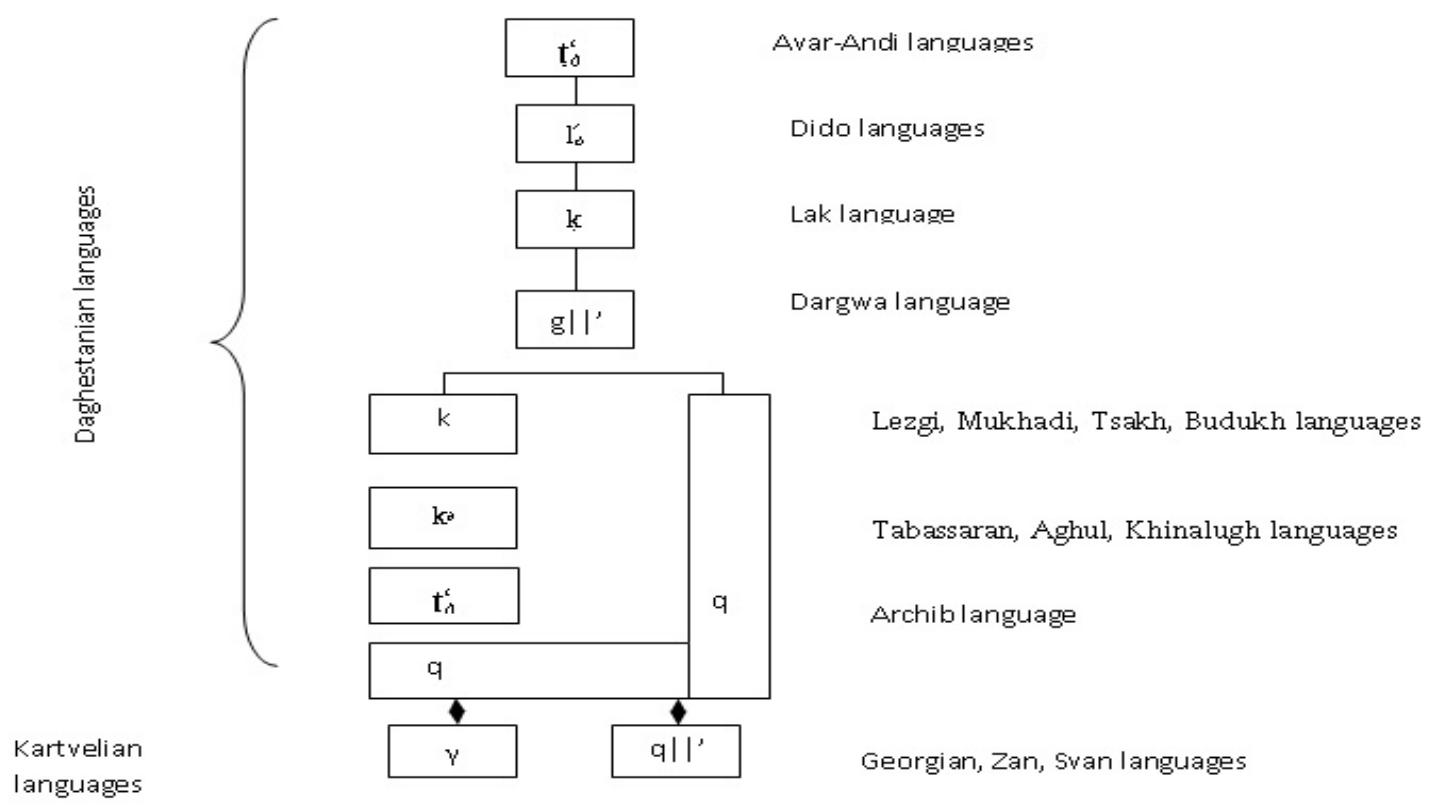


4. Comm on Daghestanian

5. Common Kartvelian

6. Common Kartvelian

$$
{ }^{*} c|| c
$$

7. Karvelian

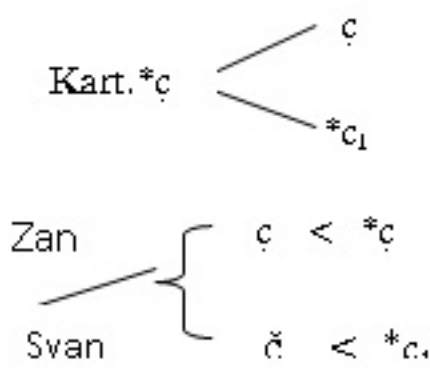

Common Kartvelian

$$
*_{\mathrm{t}||}{ }^{* s_{1} \mathrm{t}}
$$

Abkhazian

$$
\text { sól| }
$$

$*_{s_{1}} \mathrm{tw}$

Abkhazian

$\xi_{y}$

Abkhaz. 气̆

Kab. $\quad x^{-}$

Circas. $f$ 
8. Common Kartvelian

$$
{ }^{*} \mathrm{~g} w
$$

Kart. gr

Zan ' $/ q \mathrm{q}$

Syan gr

9. Common Kartvelian

$$
\begin{aligned}
& { }^{*} 3 \mid{ }^{*}{ }_{31} \\
& { }^{*} \mathrm{c} \mid{ }^{*} \mathrm{c}_{1}
\end{aligned}
$$

10. Common Kartvelian

${ }^{*} s|| *_{s_{1}}$

$*_{s}||{ }^{s t_{1}}$

${ }^{*} c||{ }^{*} c_{1}$

${ }^{*} \mathrm{q}$

${ }^{*} \mathrm{q}$
Abkhazian

$$
{ }^{*} Y_{\circ}
$$

Circas. $\gamma_{\circ}$

Abkhaz. $\quad \mathrm{V}$

Abaz. w

Basque

$c / s$

$c / s$

Basque

s

s||š(M. Kurdiani)

c/s

h

1 


\section{Basque Common Basque-Kartvelian Common Kartvelian Kart.}

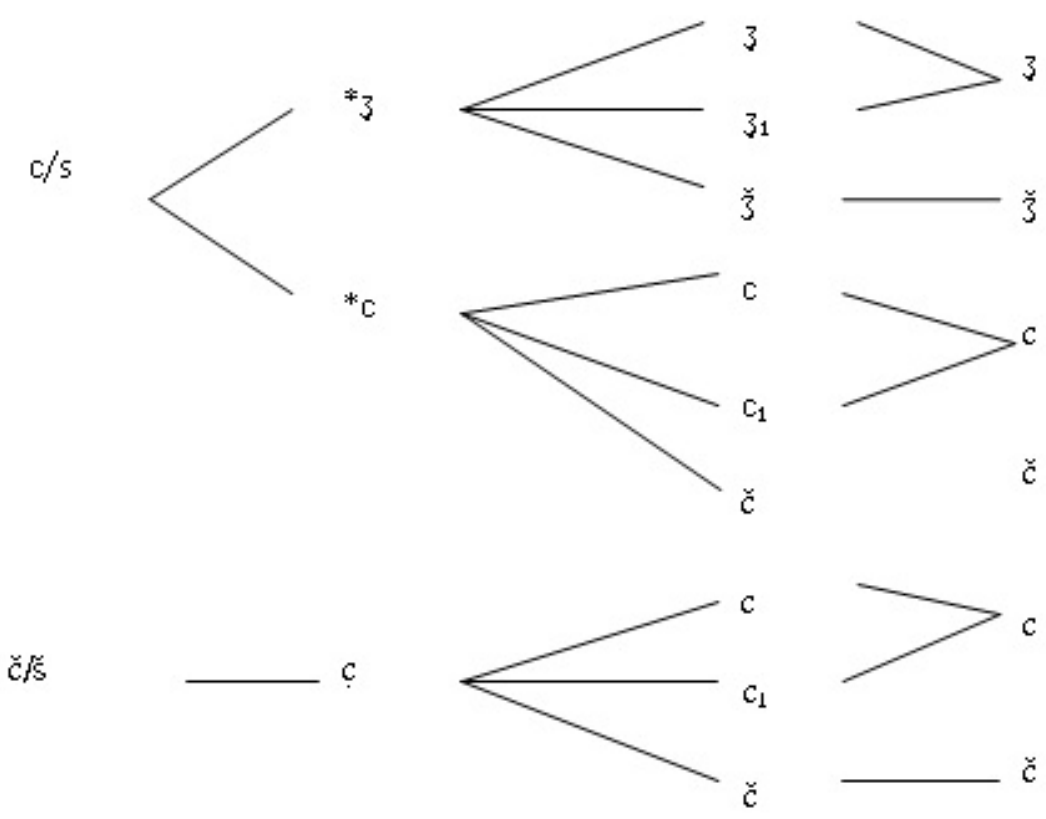

\section{For example:}

Common Daghestanian

Common-Kartvelian

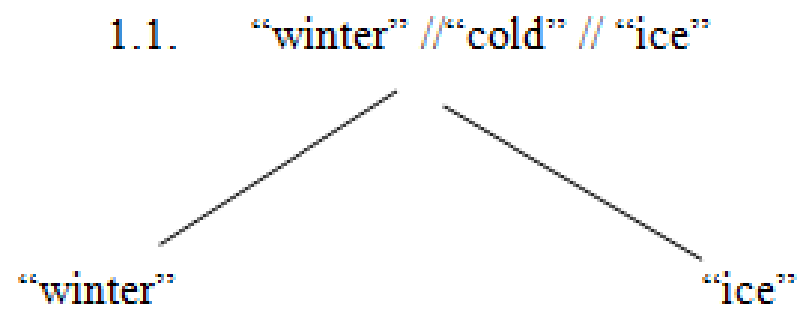

Daghestanian

Avar. in id

And. inu id.

Hun. inn id.

Darg. ini

(urax. dial.)

Lak kint id.

Did. enu id.

Hinux. eni id.

Khvarsh. enu id.

Kapuch. ine id.

"winter" // "winter" common Kartvelian

* in-w-a

Georg. in-v-a

Zan in-i //

'in- $i$ "cold"

o-in-u //

'in-u-a "ice"
Daghestanian

Darg. mi (urax.) // mi-g (cudax.) id.

Lak mi-k id.

Arch. mu- "ice”, "ice-meat"

Lezg. mur-k (erg.c. mur-k-wadi) id.

Tab. me-r- id.

Ag. me-r- "hail"

Tsax. mə-k id.

Khin. mi-k id.

"ice"

common Kartvelian

* in-el-I // * in- $\mathrm{ul}-\mathrm{i}$

Old Georg. in- el

Georg. in- ul-i

Zan . in- ur-i // 'in-er-i 
in-u-v-a $\quad$ * $^{*}$ 'in- $\mathrm{u}-\mathrm{v}-\mathrm{a}$

"winter" (Laz dial.)

1.2. Earth // Ground // Soil

Daghestanian

Avar ra "earth"

Did. ri..u "earth"

"meadow", "field"

Lezg. na..v "earth"

Buduch. no.. "soil", "clay"
Common Kartvelian *..-an-a "earth"

Georg. ..ana ; Old Georg. "earth"

" country cottage", "field"; new

Georg. "plural crops"

Zan ..ona // 'ona "plural crops",

\section{3. "love" // "want"}

Daghestanian

Common Kartvelian

Avar bo--ize

*w-ar-:

Arch. an-qes

Kartv. Georg. si-war-ul-i

Ud. bu--sun

Zan or-op-a // 'or-op-a

Common Daghestanian *

"head" // "hill top" // "upper" // "roof"

Com. Dag. * Com.Kartv. *tx

"head"

Avar. be-t-er "head"

Darg. bi-k (urax.)

// be-k (axush.)

Lak ba-k

Lezg. -il

$(<*$ wi--il)

Khin. mi-k-ir

(<*wi-k-ir)

Common Kartvelian

$$
\text { *we-3 }
$$

"mineral water",

"mineral spring"

Georg. ve za , vez-is-I (top.)

Zan men $<*$ wa-i (top.)

Common Kartvelian

*3-am-a

"brother"

Georg. 3-am-a // 3m-a

Zan ima // . uma

Svan . əm-il // . im-il

"“"brother for sister"

\section{"upper"}

Avar. t-ad

And. hi--a

Akhv. -ad-o

Did. -od-o

Lak k-a

(<*k-am-u)

"shelf"

Lezg. -w-an

Tab. k-an-u

"tube for rain"

Muxad. -aw

"roof"

Common Daghestanian

"spring"

Avar i "spring"

And. ini

Darg. $\Omega$ iniz xin. mo

Common Daghestanian

*

"brother" and "sister"

Avar wa- "brother"

ja- "sister"

dial:

Hid. wa- - ja-

Shul. va- - ja-

Toch. va-c - ja-c

And. wo--i - ja---i

Did. e-s-iju "brother" and "sister"

Darg. uzi // ui "brother" -

ruzi // rui "sister" "roof"

Avar. -om

And. -om

Did. $\quad-\mathrm{O}$

Hunz. -am-e

Lak. -am-u

"leader"

tx-um $(<*$ tx-am) "head" // "hill top" "upper"

Georg. tx-em-i

(<*txam-i)

Svan. tx-um

"head" //

ma-tx-wm-i 
(See Kurdiani 2007: 703-708).

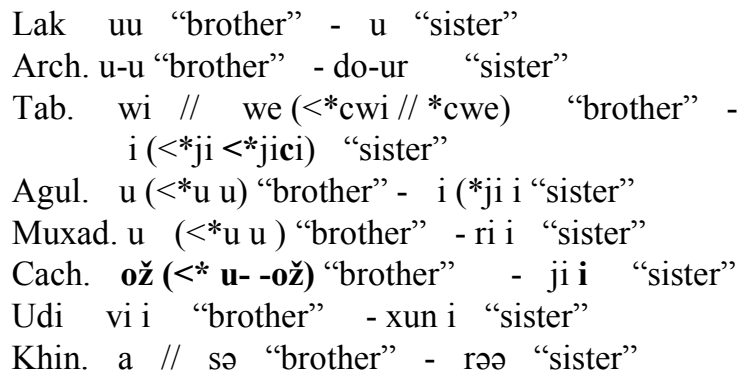

I define the next research tasks of comparative linguistics, reconstruction and synchronic description of a parent-language till differentiating, establishing an absolute chronology of a parent-language's existence, compiling of comparative phonetics and grammars of a language family, compiling of comparative and etymological dictionaries, localization of pre-mother-country of the parent-language-speaking ethnic groups and reconstruction of proto-culture.

These tasks are especially topical from the standpoint of establishing pre-historical realities of the Caucasian peoples.

\section{REFERENCES}

[1] Abdokov Aues 1983: On sound word correspondences in The North Caucasian languages, Nalchik, "Elbrus".

[2] Andronov Mikhail 1982: From the history of the classification of the Dravidian languages (theoretical basis of classification of world languages. the problem of kinship. Moscow "Nauka” (in Russian).

[3] Bomhard Alan 1990: A survey of the comparative phonology of the so-called "Nostratic" languages (trends in linguistics. Studies and Monographs 45: Linguistic change and reconstruction methodology, Berlin-New York; Mouton Gruyter" : 331-358).

[4] Bouda Karl 1949: Basque-Caucasian etymologies, Haidelberg: Carl Winter (in German).

[5] Braun Jan 1998: Euscaro-Caucasica. Historical and comparative studies on Kartvelian and Busque, Warszawa; "Dialog".

[6] Chikobava Arnold 2008: The history of the learning of the Ibero-Caucasian languages. Tbilisi, "Universal" Press (in Georgian).

[7] Chikobava Arnold 1979: Introduction to the Iberian-Caucasian linguistics. Tbilisi, Tbilisi State University Press, (in Georgian).
[8] Comrie Bernard 1998: Atlas of world's languages, Moscow, "Lik Press".

[9] Dumezil George 1933: Introduction to comparative grammar of the North Caucasian languages, Paris.

[10] Gudava Togo 1954: On the geneses of Avar Andi Dido lateral and its phonetical correspondences. (Ibero-Caucasian linguistics, VI, 55-56) (in Georgian).

[11] Ilich-Svitich Viacheslav 1971-1984: The experiense of the comparison of the in Nostratic languages (Semito-Chamit, Kartvelian, Indo-Evropian, Uralian, Dravidian, Altaian) comparative dictionary. Moscow, "Nauka" (in Russian).

[12] Khalidov 2006: Introduction to the Caucasian linguistics, Grozny, (in Russian).

[13] Kurdiani Mikheil 2007: Basics of the Iberian-Caucasian linguistics. Tbilisi, Tbilisi State University Press, (in Georgian).

[14] Lafon Rene 1948: Correspodences basque-caucasiques, II. Siffilantes et chuintanetes en basque et dans les langues caucasiques (EUSKO-JAKINTZA II: 362-367).

[15] Levin Simon 1995: Semitic and Indo-European principal etymologies (with observations on Afro-Asiatic), Amsterdam/Philadelphia: John Benjamins Publishing Company.

[16] Lomtatidze Ketevan 1955: Some issues of sound correspondences in the Iberian-Caucasian languages (on the materials of the Georgian-Abkhazian-Adygean languages) Bulletin of the Georgian Academy of Sciences, XVI, 10: 821-825) (in Georgian).

[17] Starostin Alexander 1982: Reconstruction of Pro-Enisei and interrelation of Eniseian languages ("Katian collection (anthropology, ethnography, mythology, linguistics)"), Leningrad: 144-237

[18] Trubetskoy Nikolos 1987: Collected works in philology, Moscow, "Progress".

[19] Uhlenbeck Christianus Cornelius 1909-1910: Contribution of one phonetic comparative of the dialects of Basque language ("Revista International de los Estudios Vascos, 1909, 3: 465-503; 1910, 4: 65-120) (in Spanish).

[20] Whorf Benjamin 1956: Language, thought and reality, Cambridge: Cambridge University press. 